EPJ Web of Conferences 20, 02009 (2012)

DOI: $10.1051 /$ epjconf/20122002009

(C) Owned by the authors, published by EDP Sciences, 2012

\title{
Evidence for Narrow N*(1685) Resonance
}

\author{
V. Kuznetsov ${ }^{1,2, a}$ and M.V. Polyakov ${ }^{2,3}$ \\ 1 Institute for Nuclear Research, 117312, Moscow, Russia, \\ 2 Institut für Theoretische Physik II, Ruhr-Universität Bochum, D-44780 Bochum, Germany \\ 3 Petersburg Nuclear Physics Institute, Gatchina, St. Petersburg 188350, Russia
}

\begin{abstract}
Review of experimental observations which indicate the existence of a new narrow $N^{*}(1685)$ resonance is presented.
\end{abstract}

The conventional constituent quark model $(\mathrm{CQM})$ predicts a rich spectrum of baryons resonaces [1]. In reality, almost half of the CQM-predicted nucleon and $\Delta$ resonances still escape the reliable experimental identification [2] (so-called "missing resonances"). The chiral quark soliton model ( $\chi$ QSM) is an alternative view of baryons which are treated as space/flavor rotational excitations of a classical object - a chiral mean-field. $\chi$ QSM predicts the lowest-mass multiplets of baryons to be the $1 / 2^{+}$octet and $3 / 2^{+}$decuplet - exactly as CQM does. The $\chi$ QSM predictions for higher multiplets are different from CQM [3]. Thus, the experimental study of baryon resonances provides benchmark information for the development of theoretical models and for finding relations between them.

In this context the possible observation of a new narrow resonance $N^{*}(1685)$ is of potential importance. Recently, four groups - GRAAL [4], CBELSA/TAPS[5], LNS [6], and Crystal Ball/TAPS [7], reported the evidence for a narrow structure at $W \sim 1.68 \mathrm{GeV}$ in the $\eta$ photoproduction on the neutron. The structure was observed as a bump in the quasi-free cross section and as a peak in the invariantmass spectrum of the final-state $\eta$ and the neutron $M(\eta n)$ [4,5,7] (Fig. 1,2). The width of the bump in the quasi-free cross section was found close to the smearing caused by Fermi motion of the target neutron bound in a deuteron target [4]. The width of the peaks observed in the $M(\eta, n)$ spectra was close to the instrumental resolution of the corresponding experiments $[4,5,7]$.

In Refs. [4,8-10] these observations were interpreted as a possible signal of a nucleon resonance with unusual properties: the mass near $M \sim 1.68 \mathrm{GeV}$, the narrow width, and the strong photoexcitation on the neutron. Alternatively, the authors of Refs. [11,12] explained the bump in the quasi-free $\gamma n \rightarrow \eta n$ cross section in terms of the interference of well-known resonances or as the virtual subthreshold $K \Lambda$ and $K \Sigma$ photoproduction [13]. The decisive identification of the observed structure is now a challenge for both theory and experiment.

If photoexcitation of any resonance occurs on the neutron it should generally occur also on the proton, even being suppressed by any reason. The $\eta$ photoproduction on the proton below $W \sim 1.7 \mathrm{GeV}$ is dominated by excitation of the $S_{11}(1535)$ resonance. This resonance contributes to only the $E_{0}^{+}$ multipole. The term $\left|E_{0}^{+}\right|^{2}$ is the major component of the cross section

$$
\sigma \sim\left|E_{0}^{+}\right|^{2}+\text { interference terms }
$$

while other multipoles contribute mainly through interference with the $E_{0}^{+}$. A narrow weakly-photoexcited state with the mass below $1.7 \mathrm{GeV}$ would appear in the cross section as a small peak/dip structure on the slope of the dominant $S_{11}(1535)$ resonance. On the contrary, this state may manifest itself in polarization observables due to its interference with the dominating $S$ wave.

The revision of the GRAAL beam asymmetry data for eta photoproduction on the free proton revealed a sharp resonance structure at $W=1.685 \mathrm{GeV}[14,15]$ (see also [16]). The results are shown

a e-mail: slavak@jlab.org

This is an Open Access article distributed under the terms of the Creative Commons Attribution-Noncommercial License 3.0, which permits unrestricted use, distribution, and reproduction in any noncommercial medium, provided the original work is properly cited. 

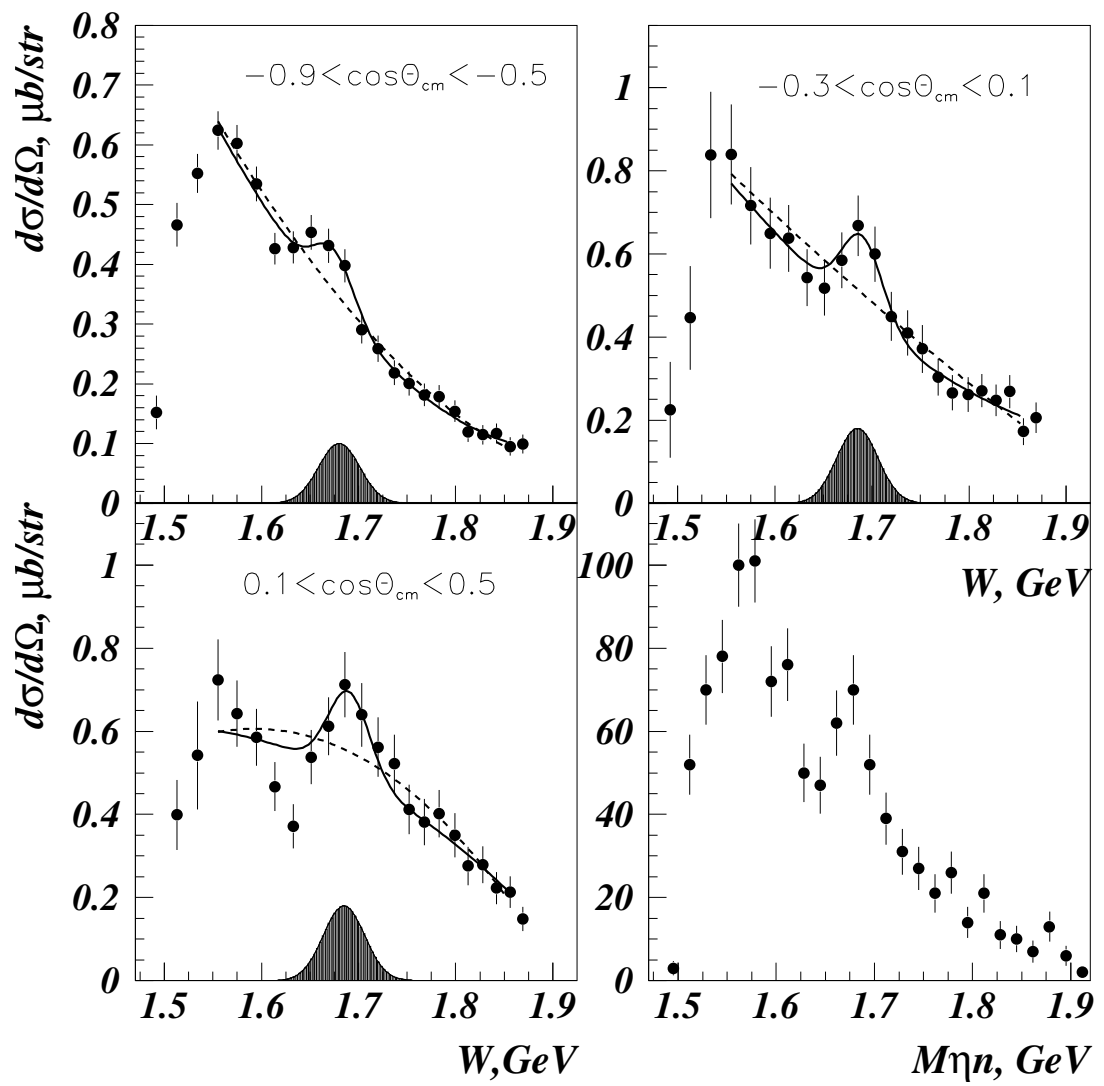

Fig. 1. Quasi-free $\gamma n \rightarrow \eta n$ cross sections and $M(\eta, n)$ spectrum from GRAAL (data from Ref. [4]).

in Fig. . Data points were obtained using fine energy bins $\Delta E_{\gamma} \sim 16 \mathrm{MeV}$. At forward angles $\left(\theta_{c m}=\right.$ $43^{\circ}$ ) and $E_{\gamma}=1.04 \mathrm{GeV}$ the data points form a sharp peak with $\Sigma$ in its maximum as large as 0.94 . The peak becomes less pronounced at $65^{\circ}$. It is replaced by an oscillating structure at $85^{\circ}$ and $106^{\circ}$. At more backward angles, the asymmetry above $1.05 \mathrm{GeV}$ drops down almost to 0 while its statistical errors grow up. The peak at forward angles and the oscillating structure at central angles together exhibit an interference pattern which may signal a narrow nucleon resonance.

The data were fit by using the multipoles of the recent E429 solution of the SAID partial-wave analysis for $\eta$ photoproduction, adding to them a narrow Breit-Wigner resonance. The narrow $S_{11}$, $P_{11}, P_{13}$, and $D_{13}$ resonances were tried one by one. Each resonance contribution was parametrized by the mass, width, photocouplings (multiplied by the square root of the $\eta N$ branching), and the phase. These parameters were varied to achieve the best agreement with experimental data. The curves with the SAID multipoles only were smooth and did not exhibit any resonant structure (Fig. ). Inclusion of a narrow resonance (either $P_{11}$, or $P_{13}$, or $D_{13}$ ) with the mass near $W \approx 1.685 \mathrm{GeV}$ allowed to reproduce the observed peak/dip structure. The mass of the included resonance was strongly constrained by experimental data. Its value belonged to the range of $M_{R}=1.686-1.690 \mathrm{GeV}$. The width was always $\Gamma \leq 25 \mathrm{GeV}$.

It is worth to add that the recent high-precision cross-section data for the $\eta$ photoproduction on the free proton from CrystalBall/TAPS revealed a small narrow dip structure at $W \sim 1.67 \mathrm{GeV}[18]$. 


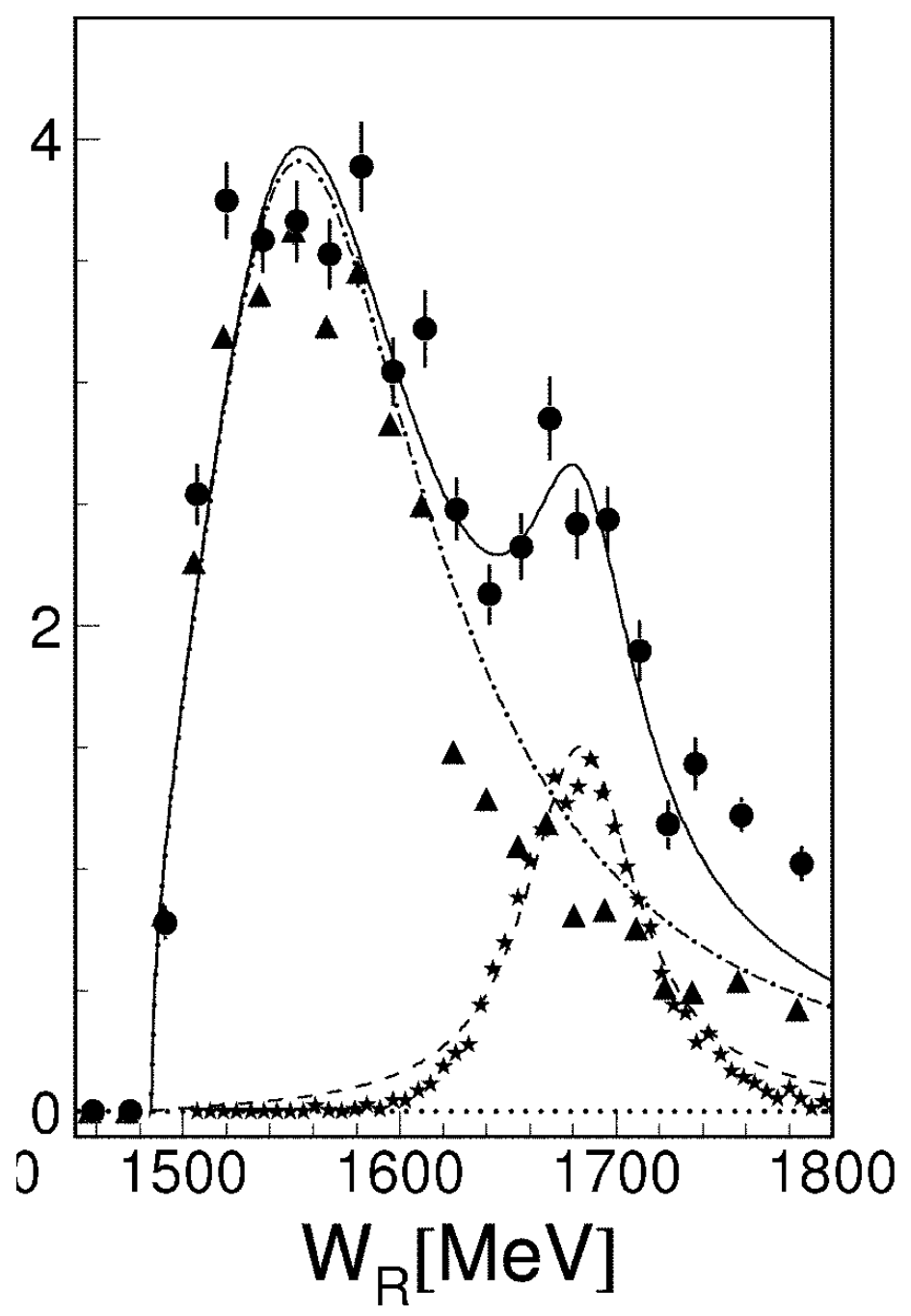

Fig. 2. $M(\eta, n)$ spectrum from CBELSA/TAPS (data from Ref. [5]).

Furthemore, if the narrow $N^{*}(1685)$ does really exist, it should be seen not only in the $\eta$ photoproduction but also in other reactions on the neutron, e.g. Compton scattering or the $\pi^{0}$ photoproduction. On the contrary, the narrow bump cannot be generated by the interference of wide resonances in these reactions, as they receive contributions of different (from the $\eta$ photoproduction) resonances.

The first study of Compton scattering on the neutron at GRAAL [19] revealed a peak at $w=1.685$ $\mathrm{GeV}$ similar to that observed in the $\eta$ photoproduction on the neutron. Fig. shows the spectrum of Compton events on the neutron. The dashed line in Fig. indicates the 2nd-order-polynomial (the background hypothesis) fit for Compton events on the neutron in the interval $W=1.585-1.888 \mathrm{GeV}$. The solid line in the same figure corresponds to the background-plus-Gaussian fit. The $\chi^{2}$ of both fits are $3.7 / 6$ and $18.5 / 9$ respectively. The log likelihood ratio of these two hypotheses $\left(\sqrt{2 \ln \left(L_{B+S} / L_{B}\right)}\right)$ corresponds to the confidence level of $\sim 4.6 \sigma$ The extracted peak position is $M=1686 \pm 7_{\text {stat }} \pm 5_{\text {syst }} \mathrm{MeV}$. and the width $\Gamma \approx 28 \pm 12 \mathrm{MeV}$. The recent very preliminary results from CrystalBall/TAPS seem to exhibit a similar peak structure [20]. Neither of such peak was observed is the $\pi^{0}$ photoproduction on the neutron $[19,21,22]$.

The combination of all experimental findings, being considered altogether, supports the existence of a narrow nucleon $N^{*}(1685)$ resonance and challenges the explanations [11,12] of the bump structure 


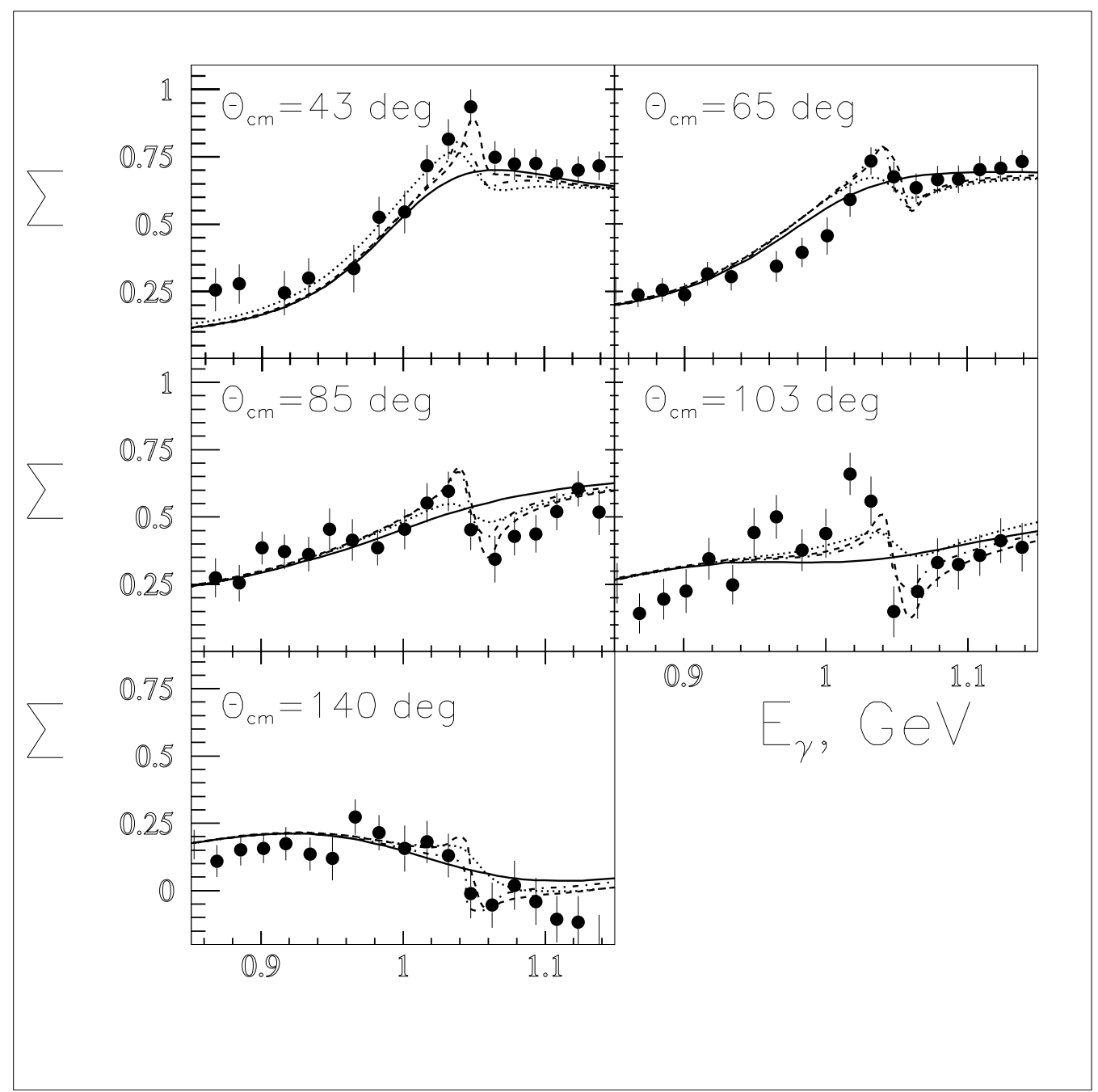

Fig. 3. Beam asymmetry $\Sigma$ for the $\eta$ photoproduction on the free proton obtained with narrow energy bins. Black circles are the data from Ref. [14]). Solid lines show our calculations based on the SAID multipoles only, dotted lines include the $P_{11}(1688)$ resonance, dashed lines are calculations with the $P_{13}(1688)$, while the dash-dotted lines use the resonance $D_{13}(1688)$.

in the quasi-free $\gamma n \rightarrow \eta n$ cross section in terms of the interference of well-known resonances. The specific features of this resonance are the narrow $(\Gamma \leq 30) \mathrm{MeV}$ width, the strong photoexcitation of the neutron, and the reduced branching ratio to the $\pi N$ final state. It is interesting that these properties are similar to those expected for the second member of the antidecuplet of extotic baryons [23].

The assumption on the virtual sub-thershold $K \Lambda$ and $K \Sigma$ photopoduction [13] cannot be exluded. However, it requires the explanation why this effect occurs in the $\gamma n \rightarrow \eta n$ and $\gamma n \rightarrow \gamma n$ reactions and is not seen in $\gamma n \rightarrow \pi^{0} n$.

\section{References}

1. S.Capstick and W.Roberts, Prog. Part. Nucl. Phys. 45, S241 (2000) .

2. C.Amsler et al., Phys. Lett. B667, 1 (2008). 


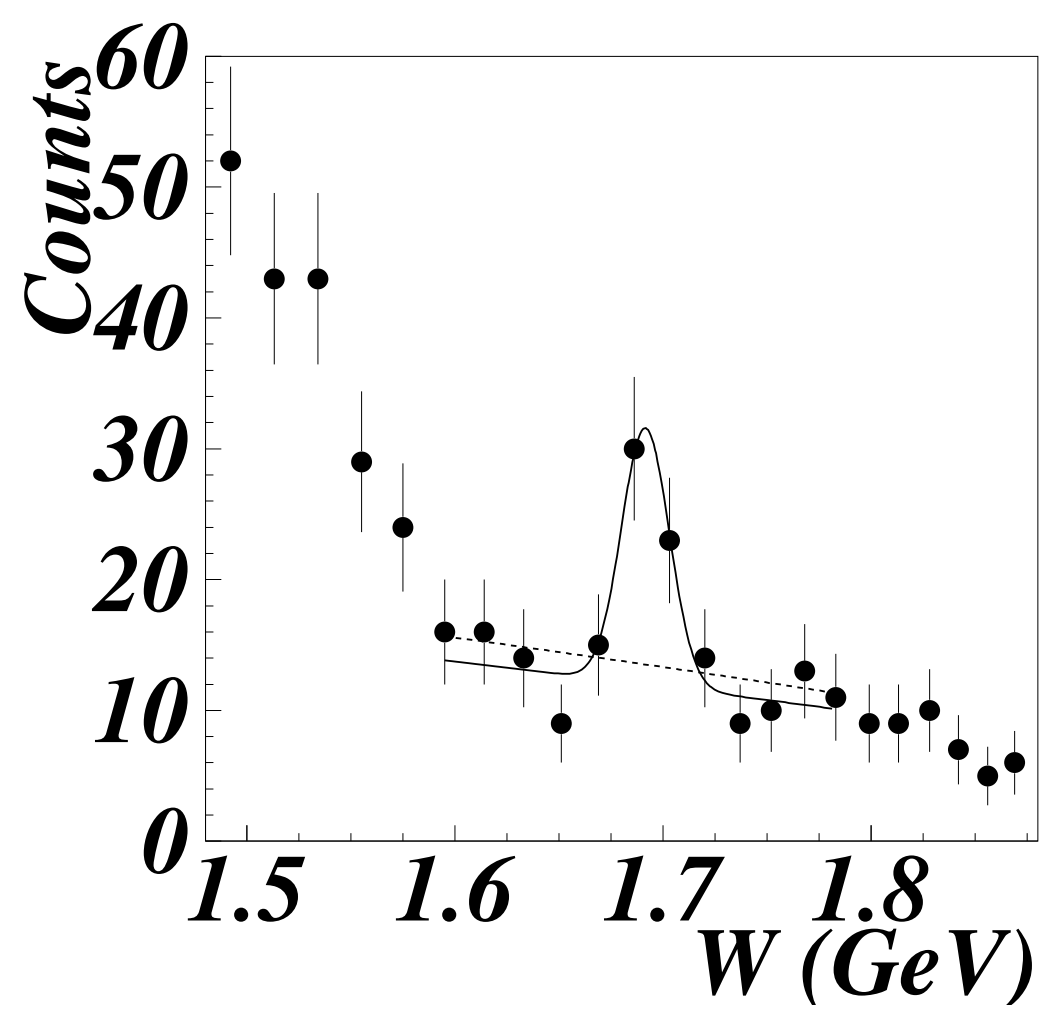

Fig. 4. The spectrum of Compton events on the neutron (data from Ref. [19]). Solid line indicates the Gaussianplus-2-order-polynomial fit. Dashed line corresponds to the 2-order-polynomial fit only.

3. D. Diakonov, V. Petrov and M. V. Polyakov, Z. Phys. A 359, 305 (1997).

4. V. Kuznetsov et al., Phys. Lett. B 647, 23 (2007).

5. I. Jaegle et al. [CBELSA Collaboration and TAPS Collaboration], Phys. Rev. Lett. 100, 252002 (2008).

6. F. Miyahara et al., Prog. Theor. Phys. Suppl. 168, 90 (2007).

7. D. Werthmuller [for the Crystal Ball/TAPS collaborations], Chin. Phys. C 33, 1345 (2009).

8. Y. I. Azimov, et al., Eur. Phys. J. A 25, 325 (2005).

9. A. Fix, L. Tiator and M. V. Polyakov, Eur. Phys. J. A 32, 311 (2007).

10. K. S. Choi, S. i. Nam, A. Hosaka and H. C. Kim, Phys. Lett. B 636, 253 (2006).

11. A. V. Anisovich et al., Eur. Phys. J. A 41, 13 (2009).

12. V. Shklyar, H. Lenske and U. Mosel, Phys. Lett. B 650, 172 (2007).

13. M. Doring and K. Nakayama, Phys. Lett. B 683, 145 (2010); M. Doring, arXiv:1010.2180 [nuclth].

14. V. Kuznetsov et al., Acta Phys. Polon. B 39, 1949 (2008).

15. V. Kuznetsov and M. V. Polyakov, JETP Lett. 88, 347 (2008).

16. It is worth to noting that the authors of Ref. [17] arrived at a different conclusion. The detailed critique of the results from Ref. [17] is available in Ref. [14]. This critique remains unreplied.

17. O. Bartalini eta al., Eur. Phys. J. A 33, 169 (2007).

18. E. F. McNicoll et al. [Crystal Ball Collaboration at MAMI], Phys. Rev. C 82 (2010) 035208.

19. V. Kuznetsov eta al., Phys. Rev. C 83, 022201(R) (2011).

20. B. Krusche, Invited seminar at Insititute of Nuclear physics, Mainz, March 2011. 


\section{EPJ Web of Conferences}

21. B. Krusche, Talk at Int. Workshop "Narrow Nucleon resonances: Predictions, Evidences, Perspectivs", June 8 - 10, 2009, Edinburg, Scotland.

22. H. Shimizu, Talk at Int. Workshop "Narrow Nucleon resonances: Predictions, Evidences, Perspectivs", June 8 - 10, 2009, Edinburgh, Scotland.

23. M. V. Polyakov and A. Rathke, Eur. Phys. J. A 18, 691 (2003). 